\title{
QUAIS SÃO OS POSSÍVEIS DETERMINANTES PARA A OCORRÊNCIA DE MORTALIDADE POR COVID-19 NAS CAPITAIS BRASILEIRA E NO DISTRITO FEDERAL?
}

\author{
WHAT ARE POSSIBLE DETERMINANTS FOR OCCURRENCE OF MORTALITY \\ BY COVID-19 FROM BRAZILIAN CAPITALS AND FEDERAL DISTRICT?
}

\author{
Thiago Ferreira de Sousa ${ }^{1}$, Sueyla Santos ${ }^{2}$, Gildeene Farias ${ }^{3}$, Gerleison Barros ${ }^{4}$, Mariana \\ Ferreira $^{5}$, Dayana Franco ${ }^{6}$
}

Universidade Federal do Recôncavo da Bahia ${ }^{1}$; Universidade Federal do Amazonas, Parintins, Amazonas, Brasil ${ }^{2}$; Faculdade Estácio de Teresina, Teresina, Piauí, Brasil ${ }^{3}$; Núcleo de Estudos em Atividade Física e Saúde (NEAFISA) da Universidade Federal do Triângulo Mineiro, Uberaba, Minas Gerais, Brasil ${ }^{4}$; Secretaria Municipal de Educação de Teresina, SEMEC ${ }^{5}$; Centro universitário de Maringá, Maringá, Paraná, Brasil.

\section{Abstract}

To estimate the determinants related to demographic, socioeconomic, behavioral, biological, perceptual indicators, cases of hospitalization and mortality from respiratory and cardiovascular disease, and health services, associated with the number of deaths by COVID19 in Brazilian capitals. Was carried out an ecological study, being a sample unit in the Brazilian capitals and the Federal District. The outcome was the numbers of accumulated cases of death by COVID-19. Linear regression used for the association. Were associated with the number of deaths by COVID-19, the number of health centers/basic health units (BHU) and hospitalization due to respiratory disease, the percentage of physically inactive $(6=0,173$; $95 \% \mathrm{Cl}=0,062 ; 0,284)$ and negative self-rated health, and cases of COVID-19 (B=0,693; IC95\%=0,291; 1,096). In conclusion, the increase in deaths due to COVID-19 in the capitals was associated with health services, physical inactivity, hospitalization from respiratory disease, negative self-rated health and number of cases of COVID-19.

Keywords: COVID-19; Mortality; Physical activity; Lifestyle; Inequalities.
Resumo

Estimar os determinantes relacionados aos indicadores demográficos, socioeconômicos, comportamentais, biológicos, perceptivos, casos de internação e mortalidade por doença respiratória e cardiovascular, e de serviços de saúde, associados ao número de óbitos por COVID-19 nas capitais brasileiras. Foi realizado um estudo ecológico, sendo a unidade amostral as capitais brasileiras e o Distrito Federal. $O$ desfecho foram os números de casos acumulados de óbito por COVID-19. Empregou-se regressão linear para a estimativa de associação. Foram associados com o aumento dos casos de óbitos por COVID-19, a quantidade de centros de saúde/unidades básicas de saúde e de internação por doença respiratória, percentual de adultos fisicamente inativos (B=0,173; IC95\%=0,062; 0,284) e de autoavaliação negativa da saúde, $e$ casos de COVID-19 (B=0,693; IC95\%=0,291; 1,096). Conclui-se que o aumento de óbitos por COVID-19 nas capitais foi associado aos serviços de saúde, inatividade física, internação por doença respiratória, autoavaliação negativa da saúde e quantidade de casos de COVID-19.

Palavras-chave: COVID-19; Mortalidade; Atividade física; Estilo de vida; Desigualdades. 
No Brasil é notório o peso do novo coronavírus sobre os casos de mortalidade, sendo mais de 260 mil óbitos no início de março de 2021 e assim representa o segundo país do mundo mais afetado pela COVID-19 ${ }^{1}$. No decorrer das observações apresentadas via painel de casos de doença pelo coronavírus 2019 (COVID-19) no Brasil pelo Ministério da Saúde ${ }^{1}$, em determinado momento houve maiores ocorrências de óbitos por COVID-19 entre as capitais do país nas regiões norte, nordeste e sudeste $^{1}$. O caso da explosão de óbitos em Manaus, capital do estado do Amazonas expôs a gravidade da epidemia em contextos de grande desigualdade social, fraca efetividade de políticas públicas e fragilidade dos serviços de saúde no Brasil $^{2}$. Elevados índices de mortalidade durante a pandemia também foram destaque entre cinco capitais brasileiras (São Paulo, Rio de Janeiro, Manaus, Fortaleza e Recife) ${ }^{1}$.

As ocorrências das altas taxas de mortalidade nas capitais do Brasil foram em decorrências também do colapso da rede pública hospitalar e precariedade na saúde pública ${ }^{2,3}$, bem como grande desigualdade social e fraca efetividade de políticas públicas, pois, estes fatores podem determinar a ocorrência de mortalidade por doença infecciosa como a COVID-19 ${ }^{2,3}$. Entretanto, é inegável que dentro de um contexto e fenômeno social iníquo como o presente, a existência do Sistema único de Saúde (SUS) é fator determinante na medida em que, ao minimizar os riscos à saúde, podem evitar um impacto proporcionalmente maior e, em situação extrema, minimizar contingentes socialmente mais vulneráveis ${ }^{4}$.

Além disso, dentre os determinantes verificados em estudos sobre os casos de óbitos relacionados ao coronavírus, a expectativa de vida demonstra moderada correlação com a mortalidade ${ }^{5}$. O Brasil por ser um país com aumento da expectativa de $v^{2} a^{6}$, tem sido impactado pela ocorrência da mortalidade por COVID-19, principalmente nas pessoas com mais de 60 anos $^{2,4}$, como também aqueles do sexo masculino $^{2}$ e também em usuários de hospitais públicos e com comorbidades ${ }^{2,4}$. Os níveis de atividades físicas regulares também se mostram associados com menor ocorrência de hospitalização por COVID-197. Além do mais, estudo realizado com 53 países da África mostrou que o aumento da quantidade de pessoal da enfermagem nos hospitais foi associado a uma diminuição dos óbitos por COVID-19, e que a falta de estrutura e capacitação no setor de

saúde estava associado a resultados ruins em relação ao agravamento da doença ${ }^{8}$.

Em função disso, conhecer melhor as características associadas ao maior número de óbitos por COVID-19 nas capitais brasileiras, poderá auxiliar no enfrentamento à doença, por meio de medidas preventivas e de controle. Deste modo poderá contribuir para minimizar os danos causados pela pandemia, assim como evitar os malefícios decorrentes de outras possíveis infecções pandêmicas. O objetivo do presente estudo foi estimar os possíveis determinantes referentes aos indicadores demográficos, socioeconômicos, comportamentais, biológicos, perceptivos, casos de morbidade e mortalidade por doença respiratória e cardiovascular, e de serviços de saúde, associados ao número de óbitos por COVID-19 nas capitais brasileiras.

\section{Metodologia}

Este é um estudo ecológico, cuja unidades de análise foram as 26 capitais dos estados brasileiros e o Distrito Federal, sendo: Aracajú, Belém, Belo Horizonte, Boa Vista, Campo Grande, Cuiabá, Curitiba, Distrito Federal, Florianópolis, Fortaleza, Goiânia, João Pessoa, Macapá, Maceió, Manaus, Natal, Palmas, Porto Alegre, Porto Velho, Recife, Rio Branco, Rio de Janeiro, Salvador, São Luís, São Paulo, Teresina e Vitória. Foi realizada a análise de dados secundários disponíveis em formato digital nas bases de dados. A descrição das bases de dados e variáveis extraídas para a investigação são apresentadas no Quadro 1. 


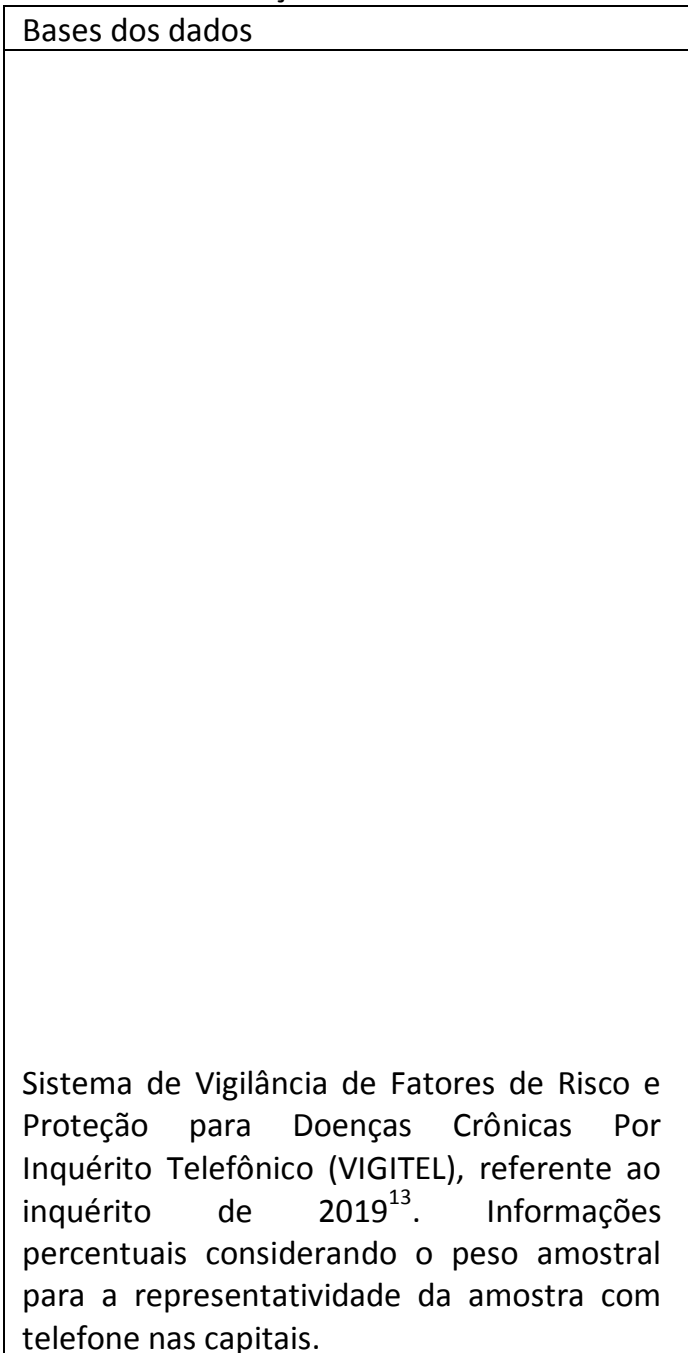

Variáveis extraídas

Percentual prática de atividade física no tempo livre (no mínimo 150 minutos semanais em atividades físicas de intensidade moderada ou pelo menos 75 minutos semanais de atividades físicas de intensidade vigorosa) no município.

Percentual de praticantes de deslocamento ativo (deslocamento para o trabalho ou escola de bicicleta ou caminhando, com pelo menos 30 minutos diários no percurso de ida e volta) no município.

Percentual de prática de atividade física insuficiente (não alcançar o equivalente a pelo menos 150 minutos semanais de atividades de intensidade moderada ou pelo menos 75 minutos semanais de atividades de intensidade vigorosa, em atividades físicas no tempo livre, no deslocamento para o trabalho/escola e na atividade ocupacional) no município.

Percentual de fisicamente inativos (não praticantes de qualquer atividade física no tempo livre nos últimos três meses, não realizar esforços físicos intensos no trabalho, não se deslocar para o trabalho ou curso/escola caminhando ou de bicicleta perfazendo um mínimo de 20 minutos no percurso de ida e volta e não ser responsável pela limpeza pesada de sua casa) no município.

Percentual de 3 horas ou mais em tempo de tela (hábito de ver ou utilizar televisão, computador, tablet ou celular por três ou mais horas/dia) no município.

Percentual de fumantes (independentemente do número de cigarros, da frequência e da duração do hábito de fumar) no município.

Percentual de consumo de frutas e hortaliças em cinco ou mais dias da semana no município.

Percentual de consumo recomendado de frutas e de hortaliças de cinco porções diárias no município.

Idade média dos participantes em anos no município.

Percentual de excesso de peso (índice de massa corporal $\geq 25 \mathrm{~kg} / \mathrm{m}^{2}$ ) no município.

Percentual de obesidade (índice de massa corporal $\geq 30$ $\mathrm{kg} / \mathrm{m}^{2}$ ) no município.

Percentual diagnóstico de hipertensão (diagnóstico médico de hipertensão arterial) no município.

Percentual de tratamento para hipertensão (diagnóstico médico, indicação de tratamento e está em uso de medicamento para controlar a pressão alta) no município.

Percentual diagnóstico de diabetes (diagnóstico médico de diabetes) no município.

Percentual de tratamento para diabetes (diagnóstico médico, indicação de tratamento e está em tratamento medicamentoso para diabetes com medicamento oral e/ou insulina) no município.

Percentual de autoavaliação negativa da saúde (avaliação do próprio estado de saúde como ruim ou muito ruim) no município. 
Continuação

Instituto Brasileiro de Geografia e Estatística ${ }^{9}$

Índice de Desenvolvimento Humano Municipal (IDH-M) referente ao ano 2010.

Percentual (\%) de esgotamento sanitário no município no ano de 2010.

Quantidade de leitos públicos e particulares para internação em estabelecimentos de saúde no ano de 2009.

Geolocalização e privacidade: os dados no Percentual médio do isolamento social entre os dias de combate à COVID-19 $9^{10}$ 01 a 07 de julho de 2020 para todo o estado.

Sistemas de Informações sobre Orçamentos Públicos em Saúde ${ }^{11}$

Valores em reais referentes aos gastos com saúde durante o ano de 2019 na atenção básica e na assistência hospitalar ambulatorial pelo município.

Instituto Trata Brasil ${ }^{12}$ Percentual de atendimento de água no município no ano de 2018

Número de casos de internação hospitalar referente a doença cardiovascular (capítulo IX, códigos 100 - 199) e respiratória (capítulo X, códigos J00 - J99) entre os meses de janeiro a junho de 2020 no município.

Número de casos de mortalidade por causas evitáveis (1.1. Reduzível pelas ações de imunoprevenção; 1.2. Reduzíveis por ações adequadas de promoção à

Ministério da Saúde, DATASUS, Sistema de saúde, prevenção, controle e atenção às doenças de causas infecciosas; 1.3.

Informação de Mortalidade (SIM), Sistema de Informação Hospitalar (SIH) e Cadastro Nacional de Estabelecimentos de Saúde $(\mathrm{CNES})^{14}$

Reduzíveis por ações adequadas de promoção à saúde, prevenção, controle e atenção às doenças não transmissíveis; 1.4. Reduzíveis por ações adequadas de prevenção,

controle e atenção às causas de morte materna) em 2019, por doença cardiovascular (capítulo IX) e respiratória (capítulo X) no município.

Quantidade de Centros de Saúde e Unidades Básicas de Saúde (UBS), como locais para realização de atendimentos de atenção básica e integral a uma população, no mês de junho de 2020.

Número de casos acumulados de pessoas com a COVID19, referentes ao dia 07 de julho de 2020 na capital e no Distrito Federal.

Site oficial do Ministérios da Saúde ${ }^{15}$

Número de óbitos acumulados de pessoas por COVID19, referentes ao dia 07 de julho de 2020 na capital e no Distrito Federal (variável dependente).

As informações foram extraídas e tabuladas no software Excel (versão 2017). Empregou-se as análises descritivas de média, desvio padrão (DP), mediana, mínimo e máximo, e informações de assimetria e curtose para a estimativa da distribuição normal, que foram considerados como aceitáveis os valores de -2 até 2 . Em função disso, observou-se que as seguintes variáveis não apresentaram distribuição normal: gastos ( $R \$$ ) na atenção básica, gastos (R\$) em atenção hospitalar ambulatorial, percentual de atendimento de água, quantidade de leitos públicos e particulares, quantidade de centros de saúde/UBS, número de casos de internação hospitalar por doença cardiovascular e por doença respiratória, percentual de tratamento para diabetes, número de casos de mortalidade evitável por doença cardiovascular e por doença respiratória, número de casos e óbitos acumulados por COVID-19. Sendo assim, recorreu-se a transformação logarítmica dos dados dessas variáveis. Para a estimativa de associação empregou-se a regressão linear simples e ajustada.

Para a análise ajustada empregou-se modelagem de análise hierárquica, considerando a hipotética relação entre as variáveis em relação ao desfecho, considerando a progressão dos níveis mais distantes (nível 1) ao nível mais 
próximo (nível 6) ${ }^{16}$. As variáveis foram assim estabelecidas:

- Nível 1, o IDH-M, o percentual de esgotamento sanitário, os gastos (R\$) em atenção básica e o percentual de atendimento de água.

- Nível 2, os percentuais de atividade física no tempo livre, deslocamento ativo, prática de atividade física insuficiente, inatividade física, 3 horas

ou mais em tempo de tela, fumantes, consumo de frutas e hortaliças/dia, consumo de 5 porções de frutas e hortaliças/dia e idade média em anos.

- Nível 3, os percentuais de excesso de peso, obesidade, diagnóstico de hipertensão, tratamento para hipertensão, diagnóstico de diabetes, tratamento para diabetes, números de

- casos de internação por doença cardiovascular e por doença respiratória.

- Nível 4, percentual de autoavaliação negativa da saúde;

- Nível 5, números de casos de mortalidade evitável por doença cardiovascular e por doença respiratória;

- $\quad$ Nível 6, número de casos acumulados de pessoas com COVID-19, quantidade de leitos públicos e particulares, a média de isolamento e os gastos (R\$) em atenção hospitalar ambulatorial.

Todas as variáveis em cada nível foram utilizadas no modelo ajustado. Adotou-se o método de seleção de variáveis backward, sendo removido em cada nível, uma por vez, as variáveis que apresentaram valor de $p>0,20$. As variáveis que permaneceram no ajuste em cada nível seguiram para o ajuste do próximo nível, não sendo mais removidos independentemente do valor de p. A análise de resíduos no nível final mostrou satisfatória homocedasticidade. Foi analisado o fator de inflação da variância (VIF) visando a identificação de multicolinearidade, e variáveis com valores $>10^{(17)}$ foram removidas independentemente do valor de $\mathrm{p}$. Empregou-se o teste de Durbin-Watson para a estimativa de autocorrelação, tendo sido considerado aceitável os valores próximos a $2^{(18)}$ e foi analisado o $R^{2}$ ajustado em cada nível. O nível de significância adotado foi de $5 \%$.

\section{Resultados}

As descrições das informações investigadas neste estudo estão apresentadas na Tabela 1. Observou-se percentual médio referente ao isolamento estadual de aproximadamente $41 \%$ (DP: 1,98), de atendimento de água em $88 \%$, esgotamento sanitário de $73 \%$. O IDH-M médio foi de 0,776 no conjunto das 26 capitais e Distrito Federal. Em relação aos comportamentos relacionados a saúde, o percentual médio de fisicamente inativos entre as capitais foi de $14,01 \%$ e de fumantes $8,32 \%$. A média de casos acumulados da COVID-19 nas capitais e Distrito Federal foi de 22.288,04 (DP: 28.867,24) e de óbitos acumulados por conta da COVID-19 foi de 1.195,11 (DP: 1.951,27). 
Tabela 1 - Descrição das variáveis explicativas das capitais brasileiras e Distrito Federal. Brasil. 2020.

\begin{tabular}{|c|c|c|c|c|c|}
\hline Variáveis & Média (DP) & Mediana & Mín. - Máx. & Assimetria & Curtose \\
\hline $\begin{array}{l}\% \text { médio de } \\
\text { isolamento }\end{array}$ & $41,07(1,98)$ & 41,24 & $35,60-45,19$ & $-0,384$ & 1,138 \\
\hline $\begin{array}{l}\text { Gastos (R\$) em } \\
A B\end{array}$ & $\begin{array}{c}386.922 .927,8 \\
(808.723 .603,1)\end{array}$ & $118.770 .610,8$ & $\begin{array}{c}23.097 .712,04- \\
4.135 .044 .948,19\end{array}$ & $0,827^{*}$ & $0,497^{*}$ \\
\hline $\begin{array}{l}\text { Gastos (R\$) em } \\
\text { AHA }\end{array}$ & $\begin{array}{c}778.693 .297,1 \\
(946.496 .034,00)\end{array}$ & $389.983 .279,30$ & $\begin{array}{c}1.040 .445,73- \\
3.669 .630 .535,21\end{array}$ & $-1,480 *$ & $2,420^{*}$ \\
\hline $\begin{array}{l}\% \text { atendimento } \\
\text { de água em } 2018\end{array}$ & $88,32(18,35)$ & 96,94 & $35,26-100,00$ & $-2,406^{*}$ & $5,114^{*}$ \\
\hline IDH-M & $0,776(0,035)$ & 0,770 & $0,721-0,847$ & 0,383 & $-0,671$ \\
\hline Quantidade de & & & & & \\
\hline $\begin{array}{ll}\text { centros } & \text { de } \\
\text { saúde/UBS } & \\
\% & \text { de }\end{array}$ & $484,74(491,64)$ & 270 & $64-2.201$ & $0,273^{*}$ & $-0,610^{*}$ \\
\hline $\begin{array}{l}\text { esgotamento } \\
\text { sanitário }\end{array}$ & $72,94(18,70)$ & 70,80 & $26,80-98,10$ & $-0,489$ & $-0,250$ \\
\hline $\begin{array}{l}\text { Idade média em } \\
\text { anos }\end{array}$ & $41,55(2,24)$ & 41,88 & $37,62-45,35$ & $-0,327$ & $-0,825$ \\
\hline $\begin{array}{l}\% \text { de } A F \text { no } \\
\text { tempo livre }\end{array}$ & $41,06(3,67)$ & 40,30 & $34,60-49,90$ & 0,439 & $-0,156$ \\
\hline $\begin{array}{l}\% \text { de prática de } \\
A F \text { insuficiente }\end{array}$ & $44,38(2,98)$ & 44,70 & $39,00-49,80$ & $-0,110$ & $-0,418$ \\
\hline $\begin{array}{l}\% \\
\text { deslocamento } \\
\text { ativo }\end{array}$ & $12,54(2,55)$ & 13,00 & $7,20-17,50$ & $-0,236$ & $-0,426$ \\
\hline $\begin{array}{l}\% \text { h ou mais em } \\
\text { tempo de tela }\end{array}$ & $61,86(2,92)$ & 62,00 & $56,90-66,50$ & $-0,034$ & $-1,193$ \\
\hline $\begin{array}{l}\% \text { de fisicamente } \\
\text { inativos }\end{array}$ & $14,01(1,93)$ & 13,60 & $10,20-17,40$ & 0,018 & $-0,364$ \\
\hline $\begin{array}{l}\% \text { de fumantes } \\
\% \text { de consumo de }\end{array}$ & $8,32(2,74)$ & 7,90 & $4,40-14,60$ & 0,596 & $-0,303$ \\
\hline $\begin{array}{l}\text { FH em cinco ou } \\
\text { mais dias } \\
\% \text { de consumo de }\end{array}$ & $33,34(6,46)$ & 32,90 & $24,30-44,60$ & 0,263 & $-1,088$ \\
\hline $\begin{array}{l}\text { cinco ou mais } \\
\text { porções de } \mathrm{FH} \\
\text { por dia }\end{array}$ & $22,21(4,33)$ & 21,70 & $15,50-31,30$ & 0,546 & $-0,574$ \\
\hline $\begin{array}{l}\% \text { de excesso de } \\
\text { peso }\end{array}$ & $54,69(2,87)$ & 54,50 & $49,10-60,90$ & 0,152 & $-0,092$ \\
\hline $\begin{array}{l}\% \text { de obesidade } \\
\% \text { diagnóstico de }\end{array}$ & $20,21(2,04)$ & 19,90 & $15,40-23,40$ & $-0,348$ & $-0,302$ \\
\hline $\begin{array}{l}\text { hipertensão } \\
\text { arterial }\end{array}$ & $23,20(3,43)$ & 24,30 & $16,90-28,50$ & $-0,177$ & $-0,927$ \\
\hline $\begin{array}{l}\% \text { de tratamento } \\
\text { para hipertensão }\end{array}$ & $81,51(5,35)$ & 81,90 & $68,80-89,70$ & $-1,017$ & 0,906 \\
\hline $\begin{array}{l}\% \text { diagnóstico de } \\
\text { diabetes }\end{array}$ & $6,77(1,10)$ & 6,80 & $4,60-8,60$ & $-0,356$ & $-0,489$ \\
\hline $\begin{array}{l}\% \text { de tratamento } \\
\text { para diabetes }\end{array}$ & $88,09(4,48)$ & 89,80 & $74,10-93,80$ & $-1,539 *$ & $2,992^{*}$ \\
\hline $\begin{array}{l}\text { № de internação } \\
\text { por doença } \\
\text { cardiovascular }\end{array}$ & $\begin{array}{c}3.687,56 \\
(5.045,29)\end{array}$ & 1.549 & $432-25.879$ & $0,367^{*}$ & $-0,607^{*}$ \\
\hline $\begin{array}{l}\text { № de internação } \\
\text { por doença } \\
\text { respiratória }\end{array}$ & $\begin{array}{c}2.908,89 \\
(4.265,46)\end{array}$ & 1.446 & $470-22.540$ & $0,576^{*}$ & $0,022^{*}$ \\
\hline
\end{tabular}




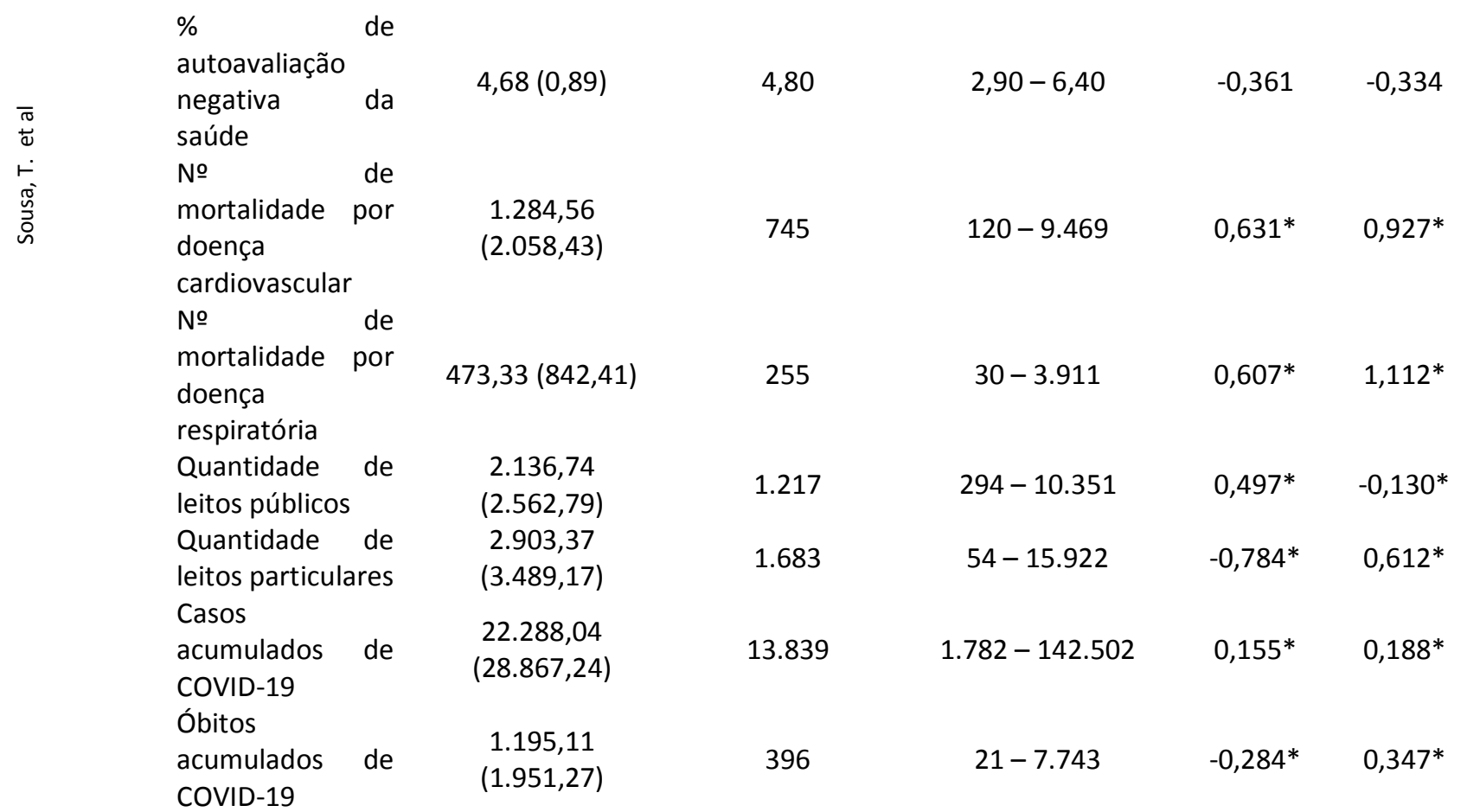

DP: Desvio padrão; Mín.: Mínimo; Máx.: Máximo; *Valores estimados considerando transformação logarítmica; IDH-M: Índice de Desenvolvimento Humano Municipal; \%: Percentual; AF: atividade física; AB: atenção básica; AHA: assistência hospitalar ambulatorial; FH: frutas e hortaliças; №: Número; SUS: Sistema Único de Saúde. 
As associações entre as variáveis explicativas e o número de casos de óbitos por COVID-19 são apresentados na Tabela 2 . Observou-se que as seguintes características foram preditoras do número de casos de óbitos por COVID-19 na regressão simples: quantidade de centros de saúde/UBS, percentuais de atividade física no tempo livre, prática de atividade física insuficiente, inatividade física, consumo de frutas e hortaliças por dia, consumo de 5 porções de frutas e hortaliças por dia, diagnóstico de diabetes, números de internações por doença cardiovascular e respiratória, percentual de autoavaliação negativa da saúde, números de mortalidade por doença cardiovascular e por doença respiratória, casos acumulados da COVID-19 e quantidades de leitos públicos e particulares.
Mostraram-se associadas a quantidade de óbitos acumulados por COVID-19 nas capitais brasileiras e Distrito Federal na regressão múltipla, o IDH-M ( $\beta$ : -15,622; IC95\%: $25,310 ;-5,934)$, a quantidade de centros de saúde/UBS ( $\beta$ : 0,796; IC95\%: 0,108; 1,484), percentual de fisicamente inativos ( $\beta$ : 0,173 ; IC95\%: 0,062; 0,284), percentual de consumo de frutas e hortaliças/dia ( $\beta$ : -0,067; IC95\%: -0,103; 0,031), número de internações por doença respiratória, percentual de autoavaliação negativa da saúde e casos acumulados de COVID19 ( $\beta$ : 0,693; IC95\%: 0,291; 1,096). Os números de mortalidade por doença cardiovascular e por doença respiratória apresentaram valores de $p$ $<0,20$, no entanto, apresentaram VIF $>10$. No nível final o valor de autocorrelação via teste Durbin-Watson foi de 2,330 e $\mathrm{R}^{2}$ ajustado de $888 \%$.

Tabela 2 - Estimativas de regressão linear simples e múltipla entre as variáveis explicativas e o número de casos de óbitos por COVID-19. Brasil. 2020.

\begin{tabular}{|c|c|c|c|c|c|c|}
\hline \multirow{2}{*}{ Variáveis } & \multicolumn{2}{|c|}{ Regressão linear simples } & \multicolumn{4}{|c|}{ Regressão linear múltipla } \\
\hline & $\beta(I C 95 \%)$ & $\mathrm{p}$ & $\beta($ IC95\%) & $\mathrm{p}$ & VIF & $\mathrm{R}^{2 \text { ajustado }}$ \\
\hline $\mathrm{IDH}_{-} \mathrm{M}^{\mathrm{a}}$ & $\begin{array}{c}-5,098(- \\
12,501 \\
2,304)\end{array}$ & 0,168 & $\begin{array}{c}-15,622(- \\
25,310 ;- \\
5,934)\end{array}$ & 0,003 & 2,716 & \\
\hline $\begin{array}{l}\text { Quantidade de centros de } \\
\text { saúde/UBS* }\end{array}$ & $\begin{array}{c}0,696(0,062 \\
1,331)\end{array}$ & 0,033 & $\begin{array}{c}0,796(0,108 \\
1,484)\end{array}$ & 0,025 & 1,673 & \\
\hline $\begin{array}{l}\% \text { de } \\
\text { sanitário }^{\text {a }}\end{array}$ & $\begin{array}{c}0,003(- \\
0,011 ; 0,018)\end{array}$ & 0,626 & $\begin{array}{c}0,016(-0,005 \\
0,038)\end{array}$ & 0,124 & 3,664 & $39,8 \%$ \\
\hline Gastos (R\$) em $A B^{*^{a}}$ & $\begin{array}{c}0,432(- \\
0,037 ; 0,901)\end{array}$ & 0,069 & $\begin{array}{c}0,037(-0,585 \\
0,659)\end{array}$ & 0,903 & 2,467 & \\
\hline$\%$ atendimento de água $*^{a}$ & $\begin{array}{c}-0,328(- \\
2,557 ; 1,900)\end{array}$ & 0,764 & $\begin{array}{c}-0,997(- \\
3,280 ; 1,287)\end{array}$ & 0,375 & 1,770 & \\
\hline$\%$ de AF no tempo livre ${ }^{b}$ & $\begin{array}{c}-0,085(- \\
0,150 ;- \\
0,020)\end{array}$ & 0,012 & $\begin{array}{c}0,036(-0,074 \\
0,145)\end{array}$ & 0,505 & 5,961 & \\
\hline$\%$ de deslocamento ativo ${ }^{b}$ & $\begin{array}{c}0,095(- \\
0,004 ; 0,193)\end{array}$ & 0,059 & $\begin{array}{c}0,019(-0,100 \\
0,138)\end{array}$ & 0,737 & 3,000 & \\
\hline $\begin{array}{l}\% \text { de prática de } A F \\
\text { insuficiente }{ }^{b}\end{array}$ & $\begin{array}{c}0,098(0,017 \\
0,179)\end{array}$ & 0,020 & $\begin{array}{c}0,009(-0,059 \\
0,077)\end{array}$ & 0,780 & 1,575 & \\
\hline$\%$ de fisicamente inativos ${ }^{b}$ & $\begin{array}{c}0,163(0,040 \\
0,286)\end{array}$ & 0,011 & $\begin{array}{c}0,173(0,062 \\
0,284)\end{array}$ & 0,004 & 1,933 & $66,5 \%$ \\
\hline $\begin{array}{l}\% \text { de } 3 \text { horas ou mais em } \\
\text { tempo de tela }{ }^{b}\end{array}$ & $\begin{array}{c}0,034(- \\
0,058 ; 0,125)\end{array}$ & 0,457 & $\begin{array}{c}0,017(-0,040 \\
0,074)\end{array}$ & 0,538 & 1,097 & \\
\hline$\%$ de fumantes ${ }^{\mathrm{b}}$ & $\begin{array}{c}-0,031(- \\
0,129 ; 0,067)\end{array}$ & 0,524 & $\begin{array}{c}-0,004(- \\
0,118 ; 0,111)\end{array}$ & 0,947 & 2,748 & \\
\hline$\%$ de consumo de $\mathrm{FH} / \mathrm{dia}^{\mathrm{b}}$ & $\begin{array}{c}-0,048(- \\
0,085 ;- \\
0,011)\end{array}$ & 0,013 & $\begin{array}{c}-0,067(- \\
0,103 ;-0,031)\end{array}$ & 0,001 & 2,244 & \\
\hline \% de consumo de 5 porções & $-0,060(-$ & 0,040 & $0,027(-0,111$ & 0,682 & 12,559 & \\
\hline
\end{tabular}




\begin{tabular}{|c|c|c|c|c|c|c|}
\hline de $\mathrm{FH} / \mathrm{dia}^{\mathrm{b}}$ & 0,118; - & & $0,166)$ & & & \\
\hline Idade média em anos ${ }^{b}$ & $\begin{array}{c}0,037(- \\
0,083 ; 0,157)\end{array}$ & 0,531 & $\begin{array}{c}-0,017(- \\
0,214 ; 0,179)\end{array}$ & 0,854 & 5,856 & \\
\hline$\%$ de excesso de peso ${ }^{c}$ & $\begin{array}{c}0,053(- \\
0,039 ; 0,144)\end{array}$ & 0,248 & $\begin{array}{l}0,041(-0,060 \\
0,141)\end{array}$ & 0,405 & 4,482 & \multirow{8}{*}{$76,0 \%$} \\
\hline$\%$ de obesidade ${ }^{c}$ & $\begin{array}{c}0,059(- \\
0,071 ; 0,190)\end{array}$ & 0,357 & $\begin{array}{c}-0,015(- \\
0,097 ; 0,067)\end{array}$ & 0,701 & 1,540 & \\
\hline $\begin{array}{l}\% \text { diagnóstico de } \\
\text { hipertensão }^{c}\end{array}$ & $\begin{array}{c}0,033(- \\
0,045 ; 0,111)\end{array}$ & 0,391 & $\begin{array}{c}0,010(-0,056 \\
0,075)\end{array}$ & 0,758 & 2,541 & \\
\hline $\begin{array}{l}\% \text { de tratamento para } \\
\text { hipertensão }^{c}\end{array}$ & $\begin{array}{c}0,014(- \\
0,036 ; 0,064)\end{array}$ & 0,574 & $\begin{array}{c}-0,006(- \\
0,044 ; 0,033)\end{array}$ & 0,762 & 1,860 & \\
\hline$\%$ diagnóstico de diabetes ${ }^{c}$ & $\begin{array}{c}0,258(0,037 \\
0,479)\end{array}$ & 0,024 & $\begin{array}{c}-0,063(- \\
0,390 ; 0,264)\end{array}$ & 0,686 & 6,102 & \\
\hline $\begin{array}{l}\% \text { de tratamento para } \\
\text { diabetes } *^{c}\end{array}$ & $\begin{array}{c}2,099(- \\
9,693 ; \\
13,891)\end{array}$ & 0,717 & $\begin{array}{c}-3,870(- \\
10,064 ; \\
2,324)\end{array}$ & 0,207 & 1,194 & \\
\hline $\begin{array}{l}\text { № de internações por } \\
\text { doença cardiovascular*c }\end{array}$ & $\begin{array}{l}0,726(0,225 \\
\quad 1,228)\end{array}$ & 0,006 & $\begin{array}{c}-0,487(- \\
1,650 ; 0,676)\end{array}$ & 0,391 & 16,922 & \\
\hline $\begin{array}{l}\text { № de internações por } \\
\text { doença respiratória* }^{c}\end{array}$ & $\begin{array}{c}0,965(0,466 \\
1,464)\end{array}$ & 0,001 & $\begin{array}{l}0,960(0,304 \\
1,616)\end{array}$ & 0,006 & 4,469 & \\
\hline $\begin{array}{l}\text { \% de autoavaliação negativa } \\
\text { da saúde }{ }^{d}\end{array}$ & $\begin{array}{l}0,421(0,174 \\
0,668)\end{array}$ & 0,002 & $\begin{array}{c}0,219(0,047 \\
0,390)\end{array}$ & 0,015 & 1,782 & $81,6 \%$ \\
\hline $\begin{array}{l}\text { № de mortalidade por } \\
\text { doença cardiovascular*e }\end{array}$ & $\begin{array}{c}1,062(0,631 \\
1,492)\end{array}$ & $<0,001$ & $\begin{array}{c}1,107(-0,061 \\
2,276)\end{array}$ & 0,062 & 24,934 & \multirow{2}{*}{$81,6 \%$} \\
\hline $\begin{array}{l}\text { № de mortalidade por } \\
\text { doença respiratória* }\end{array}$ & $\begin{array}{l}0,965(0,556 \\
1,374)\end{array}$ & $<0,001$ & $\begin{array}{c}-0,620(- \\
1,403 ; 0,162)\end{array}$ & 0,113 & 11,197 & \\
\hline $\begin{array}{l}\text { Casos acumulados de } \\
\text { COVID-19* }\end{array}$ & $\begin{array}{c}1,353(1,101 ; \\
1,605)\end{array}$ & 0,001 & $\begin{array}{c}0,693(0,291 \\
1,096)\end{array}$ & 0,002 & 3,852 & \multirow{5}{*}{$88,8 \%$} \\
\hline Média de isolamento ${ }^{f}$ & $\begin{array}{c}0,083(- \\
0,050 ; 0,215)\end{array}$ & 0,210 & $\begin{array}{c}-0,001(- \\
0,075 ; 0,072)\end{array}$ & 0,969 & 2,209 & \\
\hline Gastos (R\$) em AHA $*^{f}$ & $\begin{array}{c}0,257(- \\
0,050 ; 0,563)\end{array}$ & 0,097 & $\begin{array}{c}0,045(-0,178 \\
0,268)\end{array}$ & 0,672 & 3,889 & \\
\hline $\begin{array}{l}\text { Quantidade de leitos } \\
\text { públicos* } *^{f}\end{array}$ & $\begin{array}{c}1,152(0,682 \\
1,621)\end{array}$ & $<0,001$ & $\begin{array}{c}0,404(-0,247 \\
1,054)\end{array}$ & 0,208 & 8,726 & \\
\hline $\begin{array}{l}\text { Quantidade de leitos } \\
\text { particulares }{ }^{f}\end{array}$ & $\begin{array}{c}0,594(0,210 \\
0,977)\end{array}$ & 0,004 & $\begin{array}{c}0,125(-0,305 \\
0,555)\end{array}$ & 0,546 & 7,830 & \\
\hline
\end{tabular}

IDH-M: Índice de Desenvolvimento Humano Municipal; \%: Percentual; AF: atividade física; AB: atenção básica; AHA: assistência hospitalar e ambulatorial; FH: frutas e hortaliças; №: Número; SUS: Sistema Único de Saúde; a: nível 1; b: nível 2; c: nível 3; d: nível 4; e: nível 5; f: nível 6; *Valores estimados considerando transformação logarítmica; Teste Durbin-Watson: 2,330. 


\section{Discussão}

Neste estudo foi mostrado que as seguintes características das capitais brasileiras foram associadas ao número de óbitos por COVID-19, o IDH-M, a quantidade de centros de saúde/UBS, percentual de fisicamente inativos, percentual de consumo de frutas e hortaliças por dia, número de internações por doença respiratória, percentual de autoavaliação negativa da saúde e casos acumulados de COVID19.

Destaca-se que dentre os nossos achados, o indicador referente ao IDH-M, que congrega informações sobre longevidade, educação e renda ${ }^{19}$, mostrou-se determinante do número de óbitos por COVID-19, de modo que capitais com maiores IDH-M mostraram-se associadas com quantidades menores de mortes. Este resultado corrobora com outro estudo que mostrou em relação ao estado do Ceará, que menores valores de IDH-M foram associados com o aumento das taxas de incidência para COVID$19^{20}$. Segundo o Ministério da Saúde, o Ceará foi o terceiro estado mais afetado pela COVID-19 no Brasil entre o período de março a junho de 2020, tendo permanecido atrás apenas dos estados de São Paulo e Rio de Janeiro ${ }^{1}$. Embora os estudos tenham demonstrado que as pessoas idosas são mais acometidas pelo coronavírus, o nível educacional e principalmente renda podem favorecer como fatores de proteção, em virtude da possibilidade de acesso a melhores serviços de saúde, bem como manter-se em distanciamento físico/social ${ }^{21}$.

Outro fator que apresentou associação com o número de mortes por COVID-19, foi a maior quantidade de centros de saúde/UBS. Importante destacar que cerca de $75 \%$ da população brasileira não dispõe de plano de saúde privado, o que faz com que a grande maioria seja dependente exclusivamente do SUS $^{22}$. Com a pandemia da COVID-19 o sistema de saúde revelou-se frágil $^{23}$, em virtude do número exacerbado de pacientes, o que ocasionou colapso para o atendimento com qualidade. As UBS representam uma importante porta de entrada para o SUS, sendo assim a busca por atendimento de saúde com os primeiros sintomas de uma doença podem ocorrer nesses locais. Por outro lado, o nível primário congrega papel primordial na promoção de ações de baixa tecnologia orientadas as necessidades da saúde da população ${ }^{24}$. Sendo assim uma maior quantidade desses locais pode favorecer uma maior procura de atendimento e devido a complexidade dessa nova doença infecciosa, as ações dos profissionais das UBS podem não estar sendo adotadas pela população, como ações de higiene e distanciamento físico/social ${ }^{23}$.

Em outro extremo, este estudo não mostrou relação da quantidade de leitos em relação aos óbitos por COVID-19. Esses equipamentos compreendem a atenção em nível terciário, nos hospitais, e com o agravamento da doença a expansão do número de leitos tornouse essencial visando minimizar a quantidade de mortes pelo coronavírus ${ }^{3}$. Contudo, a não associação entre essas características neste estudo, podem estar relacionadas as características dos tratamentos clínicos, que foram alteradas ao longo dos meses em virtude do aprendizado com o cotidiano, haja vista ausência de medicamentos específicos para controlar o processo infeccioso da doença ${ }^{24}$.

Interessante mostrar que em capitais com maior percentual de fisicamente inativos, houve associação com maior quantidade de mortes por COVID-19. Em recente estudo realizado com 938 pacientes brasileiros que foram infectados pelo SARS-CoV-2, comprovados pelo exame Reverse Transcription Polymerase Chain Reaction (RT-PCR), foi mostrado que a ocorrência de internação para o tratamento da doença foi $34,3 \%$ menor entre aqueles praticantes de atividades físicas por $\geq 150$ minutos por semana, entretanto, a atividade física não apresentou proteção adicional entre os pacientes que foram hospitalizados ${ }^{7}$. Essa característica necessita de maiores esclarecimentos, possivelmente, pessoas ativas obtenham menores chances de internação hospitalar, pois apresentam uma melhor imunidade fisiológica. Face ao exposto, torna-se importante caracterizar que a prática de atividade física é essencial, um importante determinante dos níveis de saúde, o que reforça a necessidade de adoção de práticas em condições que evitem a infecção por COVID-19.

Neste estudo, a proporção de consumo de frutas e hortaliças por dia esteve associada negativamente com a ocorrência de mortalidade pela COVID-19. Uma alimentação saudável pode contribuir com o aumentar das defesas imunológicas contra o surgimento de doenças ${ }^{25}$. Por outro lado, a pandemia ocasionou impactos profundos no nível socioeconômico com o aumento do desemprego e crescimento da taxa de trabalho informal ${ }^{26}$. Eventualmente a perda de emprego ou a diminuição da renda familiar pode limitar o poder de compra de determinados grupos alimentares, como frutas e hortaliças, o que poderia justificar os achados deste estudo. 
Soma-se a isso a potencial agregação de condutas, de modo que a não adoção de um comportamento saudável pode estar vinculada a outras ações habituais em nível inadequado ${ }^{27}$.

Dentre os determinantes do aumento do número de mortes por COVID-19 apresentados neste estudo, o número de internações por doença respiratória foi um dos fatores que foi associado entre as capitais. A variedade de manifestações clínicas relacionadas as doenças respiratórias podem favorecer a ocorrência de mortalidade. Em revisão sistemática com metaanálise foi mostrado que pacientes com COVID19, na presença de doença respiratória crônica, apresentaram maiores chances de morte (OR: 2,83; IC95\%: 2,14 - 3,51), quando comparados aqueles que não possuíam a morbidade ${ }^{28}$. Por mais que fosse esperado a existência desta relação, torna-se importante observar a necessidade de prevenção e proteção da saúde para evitar o acometimento de doenças respiratórias, que podem diante disso atenuar a gravidade da evolução da COVID-19.

Foi observado que o aumento da proporção de autoavaliação negativa da saúde nas capitais foi associado como determinante da ocorrência de mortalidade por COVID-19. O julgamento de níveis negativos de saúde por uma população pode estar relacionado a outras características, como em virtude de fatores emocionais, ausência de contato com familiares, diminuição de recursos para o lazer, péssima qualidade de sono e solidão ${ }^{29}$. Autoavaliar a saúde como negativa representa um constructo preditivo de morbidade e no extremo mortalidade ${ }^{30}$. Diante disso, em capitais que houve maior percentual de pessoas que perceberam a saúde como negativa, podem representar um indicativo de problemas relacionados a um estilo de vida negativo, mas, também em detrimento de elementos socioambientais, como a dificuldade de acesso a serviços de saúde.

Este estudo mostrou que o maior número de casos estava diretamente associado com maior quantidade de mortes por COVID-19. A quantidade de casos acumulados, juntamente com outras características explicam $88,8 \%$ dos óbitos pelo coronavírus nas capitais. No Brasil, medidas em nível municipal e estadual foram adotadas para diminuir o contágio da COVID-19, entretanto, muitos casos surgiram diariamente ${ }^{31}$ e um dos maiores desafios representam as pessoas assintomáticas e pré-sintomáticas ${ }^{32}$.

Desde os primeiros relatos no final de 2019, essa doença propagou-se rapidamente entre as pessoas, expandindo pelo contágio acelerado e levando a uma pandemia ${ }^{33}$. Grandes incertezas surgiram referente a compreensão da disseminação da COVID-19 ${ }^{33}$. Nesta situação, desde o dia 7 de fevereiro de 2020, por meio da Lei $n$ o 13.979/2020, que dispõe sobre as medidas para enfrentamento da COVID-19 no país, estados e seus municípios, implementaram restrições sanitárias e intervenções não farmacológicas como uso de máscara facial e distanciamento social ${ }^{31}$.

Desta forma a necessidade de distanciamento físico/social e evitar aglomerações tornaram-se ações fundamentais para minimizar o número de casos, e possíveis internações hospitalares, que podem resultar em óbitos $^{34}$. O painel de casos de doença pelo coronavírus 2019 no Brasil pelo Ministério da Saúde até o dia 04 de março de 2021, mostrou a ocorrência de 10.793 .732 casos confirmados, com taxa de letalidade de $2,4 \%$ e marca de 260.970 mortes $^{1}$.

Este estudo apresenta limitações que devem ser consideradas, como o fato das informações de casos e óbitos por COVID-19 ter sido obtidas do período de ascensão da doença no ano de 2020, normalmente reconhecido com "primeira onda". Desde então, houve modificações nesses números ao longo do tempo. Além disso, a obtenção das informações por meio de dados secundários disponibilizados em diferentes sistemas podem apresentar ausência de atualização em tempo real. Contudo, como ferramenta de divulgação das características populacionais congregam grau de relevância e importância para gestores nas tomadas de decisões.

Possíveis limitações são derivadas das características específicas dos dados, como informações ponderadas para representar a população de cada capital provenientes do sistema VIGITEL, variação de idade ampla, como nas informações relativas às internações por doença e mortalidade via DATASUS, o uso de dados percentuais do isolamento social do estado como uma aproximação de valores ocorridos especificamente na capital e o emprego de dados referentes a informações de anos anteriores, como o IDH-M provenientes do censo de 2010. Embora a ocorrência de falácia ecológica, por representar informações das capitais, este estudo apresenta pontos positivos, pois inclui possíveis determinantes da população, considerando elementos individuais $e$ socioambientais. 


\section{Considerações finais}

Conclui-se que foram associados com

maior ocorrência de óbitos por COVID-19, as capitais que apresentaram maior quantidade de centros de sáude/UBS, maior percentual de adultos fisicamente inativos, maior número de internações por doença respiratória, maior percentual de autoavaliação negativa da saúde e maior quantidade de casos acumulados de COVID-19. Em contrapartida, foram associados com menor ocorrência de óbitos por COVID-19 as capitais com maior IDH-M e com maior percentual de consumo de frutas e hortaliças por dia.

\section{Referências}

1. Brasil. Ministério da Saúde. Painel de casos de doença pelo coronavírus 2019 (COVID19) no Brasil pelo Ministério da Saúde [Internet]. 2021 [citado 2021 Mar 4]. Disponível em: https://covid.saude.gov.br/

2. Orellana JDY, Cunha GM, Marrero L, Horta LB, Leite IC. Explosão da mortalidade no epicentro amazônico da epidemia de COVID-19. Cad Saúde Pública. 2020;36(7):e00120020. https://doi.org/10.1590/0102-311X00120020

$3 . \quad N o r o n h a$ KVMS, Guedes GR, Turra CM, Andrade MV, Botega L, Nogueira D, et al. Pandemia por COVID-19 no Brasil: análise da demanda e da oferta de leitos hospitalares e equipamentos de ventilação assistida segundo diferentes cenários. Cad Saúde Pública. 2020;36(6):e00115320.

https://doi.org/10.1590/0102-311X00115320

$4 . \quad$ Maciel EL, Jabor P, Goncalves JE, TristãoSá R, Lima RCD, Reis-Santos B, et al. Factors associated with COVID-19 hospital deaths in Espírito Santo, Brazil, 2020. Epidemiol Serv Saúde.

2020;29(4):e2020413.

https://doi.org/10.1590/s167949742020000400022

5. Hallal PC. Worldwide differences in COVID-19-related mortality. Ciênc saúde coletiva. 2020;25(Suppl 1):2403-2410. https://doi.org/10.1590/141381232020256.1 .11112020

6. Brasil. Ministério da Saúde. Ministério da Mulher, da Família e dos Direitos Humanos. Expectativa de vida do brasileiro sobe para 74,6 anos [Internet]. 2018. [acessado em 1 mar. 2021]. Disponível em: https://www.gov.br/mdh/pt$\mathrm{br} / \mathrm{sdh} /$ noticias/2013/dezembro/expectativa-devida-do-brasileiro-sobe-para-74-6-anos

7. Souza FR, Motta-Santos D, Soares DS,
Lima JB, Cardozo GG, Guimarães LSP, et al. Physical Activity Decreases the Prevalence of COVID-19-associated Hospitalization: Brazil EXTRA Study. medRxiv [Internet]. 2020. [access on 6 fev. 2020]. Available from: https://www.medrxiv.org/content/10.1101/2020 $.10 .14 .20212704 v 1$. full.pdf

8. Okeahalam C, Williams V, Otwombe K. Factors associated with COVID-19 infections and mortality in Africa: a cross-sectional study using publicly available data. BMJ Open. 2020;10(11):e042750.

https://doi.org/10.1136/bmjopen-2020-042750 9. Instituto Brasileiro de Geografia e Estatística. Panoramas das cidades do Brasil 2010 [Internet]. Rio de Janeiro: Instituto Brasileiro de Geografia e Estatística; 2010 [acessado em 28 dez. 2020]. Disponível em: https://cidades.ibge.gov.br/brasil/panorama

10. Inloco I. Geolocalização e privacidade: os dados no combate à COVID-19 | Inloco [Internet]. 2020 [acessado em 27 dez. 2020]. Disponível em: https://mapabrasileirodacovid.inloco.com.br/pt/ 11. Brasil. Ministério da Saúde. Sistemas de informações sobre orçamentos públicos em saúde: Consulta de lei de responsabilidade fiscal. Brasília: Ministério da Saúde; 2019 [acessado em 27 dez. 2020]. Disponível em: http://siops.datasus.gov.br/consleirespfiscal.php

12. Brasil. Ministério das Cidades. Instituto Trata Brasil: Ranking do saneamento, 2020 [Internet]. 2020 [acessado em 27 dez. 2020]. Disponível em: http://www.tratabrasil.org.br/estudos/estudositb/itb/ranking-do-saneamento-2020

13. Brasil. Ministério da Saúde. Secretaria de Vigilância em Saúde. Departamento de Vigilância de Doenças e Agravos não Transmissíveis e Promoção de Saúde. VIGITEL Brasil 2019: Vigilância de fatores de risco e proteção para doenças crônicas por inquérito telefônico. Brasília: Ministério da Saúde; 2019.

14. DATASUS. DATASUS: tecnologia da informação a serviço do SUS [Internet]. [acessado em 22 abr. 2021]. Disponível em: http://www2.datasus.gov.br/DATASUS/index.ph p?area $=02$

15. Brasil. Ministério da Saúde. Covid-19 no Brasil: Casos e óbitos, 2020 [Internet]. 2020 [acessado em 30 dez. 2020]. Disponível em: https://susanalitico.saude.gov.br/extensions/covi d-19 html/covid-19 html.html

16. Victora CG, Huttly SR, Fuchs SC, Olinto MT. The role of conceptual frameworks in epidemiological analysis: a hierarchical approach. Int J Epidemiol. 1997;26(1):224-7. 
https://doi.org/10.1093/ije/26.1.224

17. Akinwande MO, Dikko HG, Samson A. Variance inflation factor: as a condition for the inclusion of suppressor variable(s) in regression analysis. Open J Stat. 2015;5(7):754-67. http://dx.doi.org/10.4236/ojs.2015.57075

18. Durbin J, Watson GS. Testing for serial correlation in least squares regression, II. Biometrika. 1951;38(1-2):159-79. https://doi.org/10.1093/biomet/38.1-2.159

19. Programa das Nações Unidas Para o Desenvolvimento. Atlas do Desenvolvimento Humano no Brasil [Internet]. 2019 [acessado em 30 dez. 2020]. Disponível em: http://www.atlasbrasil.org.br/acervo/atlas

20. Romero JAR, Silva FAM. Relação entre as condições socioeconômicas e a incidência da pandemia da COVID-19 nos municípios do Ceará. Revista UFRR/BOCA. 2020;3(7):85-95. https://doi.org/10.5281/zenodo.3923443

21. Jardim VC, Buckeridge MS. Análise sistêmica do município de São Paulo e suas implicações para o avanço dos casos de Covid-19. Estud Av. 2020;34(99):157-174. https://doi.org/10.1590/s01034014.2020.3499.010

22. Castro MC, Massuda A, Almeida G, Menezes-Filho NA, Andrade MV, Noronha KVMS, et al. Brazil's unified health system: the first 30 years and prospects for the future. Lancet. 2019;394(10195):345-356.

https://doi.org/10.1016/S0140-6736(19)31243-7

23. Vitória AM, Campos GWS. Só com APS forte o sistema pode ser capaz de achatar a curva de crescimento da pandemia e garantir suficiência de leitos UTI. COSEMS/SP, 2020. [acessado em 28 abr. 2021]. Disponível em: https://www.cosemssp.org.br/wp-

content/uploads/2020/04/So-APS-forte-para-terleitos-UTI-.pdf

24. Engstrom $E$, Melo $E$, Giovanella $L$, Mendes A, Grabois V, Mendonça MHM. Recomendações para a organização da Atenção Primária à Saúde no SUS no enfrentamento da Covid-19. [acessado em 28 abr. 2021]. Disponível em:

https://www.arca.fiocruz.br/bitstream/icict/4140 4/2/RecomendacoesAPSEnfrentamentoCovid19.pdf

25. Steele EM, Rauber F, Costa CS, Leite MA, Gabe KT, Louzada MLC, et al. Dietary changes in the NutriNet Brasil cohort during the covid-19 pandemic. Rev Saúde Pública. 2020;54. https://doi.org/10.11606/s15188787.2020054002950

26. Costa SS. The pandemic and the labor market in Brazil. Rev Adm Pública.
2020;54(4):969-978.

https://doi.org/10.1590/0034-761220200170x

27. Bezerra ACV, Silva CEM, Soares FRG, Silva JAM. Factors associated with people's behavior in social isolation during the COVID-19 pandemic. Ciênc saúde coletiva. 2020;25(Supl.1):2411-2421.

https://doi.org/10.1590/1413$\underline{81232020256.1 .10792020}$

28. Degarege A, Naveed Z, Kabayundo J, Brett-Major D. Risk factors for severe illness and death in COVID-19: a systematic review and meta-analysis. medRxiv [Internet]. 2020 [access on 22 jan. 2020]. Available from: https://www.medrxiv.org/content/10.1101/2020 $.12 .03 .20243659 \mathrm{v} 1$

29. Losada-Baltar A, Jiménez-Gonzalo L, Gallego-Alberto L, Pedroso-Chaparro M del S, Fernandes-Pires J, Márquez-González M. "We Are Staying at Home." Association of Self-perceptions of Aging, Personal and Family Resources, and Loneliness With Psychological Distress During the Lock-Down Period of COVID-19. J Gerontol B Psychol Sci Soc Sci. 2021;76(2):e10-e16. https://doi.org/10.1093/geronb/gbaa048

30. Bopp M, Bopp C, Schuchert M. Active Transportation to and on Campus is Associated With Objectively Measured Fitness Outcomes Among College Students. J Phys Act Health. 2015;12(3):418-23.

https://doi.org/10.1123/ipah.2013-0332

31. Aquino EML, Silveira IH, Pescarini JM, Aquino R, Souza-Filho JA, Rocha AS, et al. Medidas de distanciamento social no controle da pandemia de COVID-19: potenciais impactos e desafios no Brasil. Ciênc saúde coletiva. 2020;25(Suppl.1):2423-2446.

https://doi.org/10.1590/141381232020256.1 .10502020

32. Kimball A, Hatfield KM, Arons M, James A, Taylor J, Spicer K, et al. Asymptomatic and Presymptomatic SARS-CoV-2 Infections in Residents of a Long-Term Care Skilled Nursing Facility - King County, Washington, March 2020. MMWR Morb Mortal Wkly Rep. 2020;69(13):377381. https://doi.org/10.15585/mmwr.mm6913e1 33. Gandhi RT, Lynch JB, Del Rio C. Mild or Moderate Covid-19. N Engl J Med. 2020;383(18):1757-1766.

https://doi.org/10.1056/NEJMcp2009249

34 .Andrade CLT, Pereira CCA, Martins M, Lima SML, Portela MC. COVID-19 hospitalizations in Brazil's Unified Health System (SUS). PLOS ONE. 2020; 15(12):e0243126. https://doi.org/10.1371/journal.pone.0243126 


\section{Endereço para Correspondência}

Thiago Ferreira de Sousa

Universidade Federal do Recôncavo da Bahia.

Centro de Formação de Professores.

Avenida Nestor de Mello Pita, no 535 - Número

da caixa postal: 64 , Centro

Amargosa - Bahia - Brasil

CEP: $45.300-000$

E-mail: tfsousa_thiago@yahoo.com.br

Recebido em 19/03/2021

Aprovado em 01/05/2021

Publicado em 15/06/2021 